\title{
Differential diagnosis of thalassemia and iron deficiency anemia in pregnant women using new formulas from multidimensional analysis of red blood cells
}

\author{
Haijun Xiao $^{1 \#}$, Yidan Wang ${ }^{1 \#}$, Yi Ye ${ }^{2}$, Chen Yang ${ }^{1}$, Xiaolong $\mathrm{Wu}^{2}$, Xiurong $\mathrm{Wu}^{1}$, Xiaomei Zhang ${ }^{2}$, \\ Tianxi $\mathrm{Li}^{1}$, Jianping $\mathrm{Xiao}^{2}$, Ling Zhuang ${ }^{1}$, Huan $\mathrm{Qi}^{2}$, Feng Wang ${ }^{1}$ \\ ${ }^{1}$ Department of Medical Laboratory, Shenzhen Baoan Women's and Children's Hospital, Shenzhen, China; ${ }^{2}$ Hematology Application and Research \\ Department, Shenzhen Mindray Bio-Medical Electronic Co., Ltd, Shenzhen, China \\ Contributions: (I) Conception and design: H Xiao, J Xiao, Y Ye; (II) Administrative support: None; (III) Provision of study materials or patients: None; \\ (IV) Collection and assembly of data: X Wu, X Wu, C Yang, T Li, L Zhuang; (V) Data analysis and interpretation: F Wang, H Qi, X Zhang, X Wu; (VI) \\ Manuscript writing: All authors; (VII) Final approval of manuscript: All authors. \\ \#These authors contributed equally to this work. \\ Correspondence to: Feng Wang. Department of Medical Laboratory, Shenzhen Baoan Women's and Children's Hospital, Shenzhen, China. \\ Email: wangfengnew@qq.com; Huan Qi. Hematology Application and Research Department, Shenzhen Mindray Bio-Medical Electronic Co., Ltd, \\ Shenzhen, China. Email: qihuan@mindray.com.
}

Background: Iron deficiency anemia (IDA) and thalassemia trait (TT) are the most common conditions of microcytic hypochromic anemia (MHA) in pregnant women. We used the BC-6800Plus analyzer to study the utility of erythrocyte and reticulocyte parameters for distinguishing TT from IDA in pregnant women.

Methods: A total of 454 anemic pregnant women, including 340 with IDA, 66 with $\beta$-thalassemia trait $(\beta$-TT) and 48 with $\alpha$-thalassemia trait $(\alpha$-TT), were included. Multiple comparisons among groups were performed, and diagnostic performance of parameters was determined using receiver operating characteristic (ROC) curve analysis, with $\mathrm{P}<0.05$ indicating statistical significance.

Results: Reticulocyte production index (RPI) and the average volume of mature red blood cells $(\mathrm{MCVm})$ in the IDA group were significantly higher than in the $\beta$-TT and $\alpha$-TT groups. Red blood cell (RBC), reticulocyte percentage (Ret\%), and RPI in the IDA group were significantly lower than in the $\alpha$-TT and $\beta$-TT groups. We devised MHA $1=0.42 \times \mathrm{MCH}-0.57 \times \mathrm{RPI}-0.08 \times \% \mathrm{MICROr}-9.38$ to distinguish IDA from $\alpha$-TT. With a cut-off value of 0.61 , the area under the receiver operating characteristic curve (AUC), sensitivity, and specificity were $0.868,90.9 \%$, and $68.5 \%$, respectively. We devised MHA $2=0.04 \times \%$ MICROr +0.12 $\times$ MCVm $-13.76 \times$ Ret\# -6.29 to distinguish IDA from $\beta$-TT. With a cut-off value of 0.55 , the AUC, sensitivity, and specificity were $0.878,81.3 \%$, and $80.3 \%$, respectively.

Conclusions: Erythrocyte indices and formulas can be used as initial methods for the differential diagnosis of TT and IDA. MHA 1 and MHA 2 were the most useful indices in the differential diagnosis of $\alpha$-TT from IDA and $\beta$-TT from IDA in pregnant women.

Keywords: Microcytic hypochromic anemia (MHA); iron deficiency anemia (IDA); thalassemia; diagnostic performance; multidimensional analysis of red blood cell (multidimensional analysis of RBC).

Submitted Nov 09, 2020. Accepted for publication Jan 06, 2021.

doi: 10.21037/atm-20-7896

View this article at: http://dx.doi.org/10.21037/atm-20-7896 


\section{Introduction}

Iron deficiency anemia (IDA) and thalassemia trait (TT) are the most common conditions of microcytic hypochromic anemia (MHA). IDA is the most common complication during gestation. The estimated occurrence rate is $30 \%$ among non-pregnant women and more than $42 \%$ among pregnant women $(1,2)$. Thalassemia is a common monogenic disease caused by defects in the synthesis of hemoglobin chains (3), and is highly prevalent in the tropics and subtropics (4). In China, the incidence rate of thalassemia mutations along the coastal areas of southern China is higher than that in inland regions $(3,5)$. Therefore, in Guangdong Province, the identification of thalassemia carriers is important in premarital counselling to prevent the inheritance of severe and potentially lethal forms of thalassemia (6). For the diagnosis of thalassemia, a complete blood count is first performed. Then, for confirmation of the thalassemia diagnosis, a hemoglobin and/or genetic analysis is required, and an analysis of iron status parameters is needed to confirm IDA. However, these confirmatory assays are expensive, and the process is complex. Thus, to improve the accuracy of further diagnostic tests, TT and IDA can be preliminarily identified more accurately and effectively through a complete blood count, to avoid unnecessary iron therapy in thalassemia carriers or to provide an indication for iron supplementation in IDA patients (7). Furthermore, this approach could facilitate the rational allocation of health-care resources, as well as early and accurate treatment interventions.

The Mindray BC-6800Plus analyzer applies the Mie theory in the RET channel, and the measured forward scatter (FSC) signal and side scatter (SSC) signal (SF-Cube technology) for each red blood cell (RBC) are converted into the volume and hemoglobin concentration (HC). The $\mathrm{HC}$ of the sample is used as the $\mathrm{X}$-axis, and the volume (VOL) is used as the $\mathrm{Y}$-axis to draw a scatter diagram (VHF map). The RBC VHF map shows the different characteristics of erythrocytes and reticulocytes one by one, and may be useful in the differential diagnosis of MHA.

In recent decades, erythrocyte indices and the various formulas derived from them have been used to distinguish TT from IDA in adults or children (8-29). However, few hematological indices have shown significance for the screening of pregnant women with TT. Therefore, the aim of the present study was to distinguish TT from IDA in pregnant women based on SF-Cube technology in order to obtain more detailed signal characteristics of mature RBCs and reticulocytes.

We present the following article in accordance with the STARD reporting checklist (available at http://dx.doi. org/10.21037/atm-20-7896).

\section{Methods}

\section{Study subjects}

The study was conducted at the Department of Medical Laboratory of Shenzhen Baoan Women's and Children's Hospital from July 26 to December 13, 2019. All samples were obtained from pregnant women with mean corpuscular volume $(\mathrm{MCV})<80 \mathrm{fL}$ and/or mean corpuscular hemoglobin $(\mathrm{MCH})<27 \mathrm{~g} / \mathrm{L}$ from routine laboratory tests. Every patient contributed only a single sample to the study. This practice is in accordance with laboratory standard operating procedures (SOPs). The diagnosis of each patient was retrieved from the hospital information system (HIS) based on their laboratory test results and medical records. We excluded samples from patients with infection or inflammatory disease, patients who were taking iron tablets at the time of sample collection or had taken iron tablets during the previous 3 months, and patients who had received a blood transfusion in the preceding 3 months. This study was approved by Ethics Committee of Shenzhen Baoan Women's and Children's Hospital (LLSCHY 201910-15-04), and informed consent was not required because only residual samples were collected and tested in this study. All procedures performed in this study involving human participants were in accordance with the Declaration of Helsinki (as revised in 2013). Pregnant women were defined as having IDA when serum ferritin (SF) was $<15 \mathrm{~g} / \mathrm{L}$ and hemoglobin was $<110 \mathrm{~g} / \mathrm{L}$ (30). $\beta$-TT was confirmed by polymerase chain reaction-reverse dot blot (PCR-RDB) analysis for the most common beta gene mutations, $\alpha$-TT was detected by gap-PCR analysis for the most common alpha gene deletions, and point mutations were detected using PCR-RDB.

Finally, data from 452 pregnant women with MHA were collected. Based on their diagnostic information, patients were classified into one of the following groups: IDA ( $n=340$, $58.1 \%)$, with an age range of 19-41 years and gestational age range of $22-41$ weeks; $\beta$-TT $(n=48,17.6 \%)$, with an age range of 22-41 years and a gestational age range of first trimester-41 weeks; and $\alpha$-TT trait $(n=66,24.3 \%)$, with an age range of $22-41$ years and gestational age range of first trimester-40 weeks. The mean HbA2 level in the $\beta$-TT 
group was $5.2 \% \pm 0.3 \%$ (min-max: $4.7 \%$ to $5.8 \%$ ). The $\beta$-TT group contained 48 cases, including 1 case of CDs14-15, 3 cases of CD17, 1 case of CD28, 14 cases of CDs41-42, 11 cases of CD654, 2 cases of CD71-72, and 16 others. In the $\alpha$-TT group, 6 patients had single-gene deletions ( 4 had $\alpha 3.7$ deletions, 1 had $\alpha 4.2$ deletions, and 1 had SEA deletions), 45 had double-gene deletions ( 2 had heterozygote $\alpha 3.7$ deletions, 2 had heterozygote $\alpha 4.2$ deletions, and 41 had heterozygote $S E A$ deletions), and 15 had non-deletional mutations.

\section{Analytical methods}

Venous blood samples were drawn into evacuated tubes containing K2-EDTA, kept at room temperature and processed within $6 \mathrm{~h}$ from the time of blood collection. The complete blood count analysis was performed using the Mindray BC-6800Plus automated hematology analyzer (Mindray Corporation, Shenzhen, China), which was calibrated, controlled, and maintained according to the manufacturer's recommendations. RPI represents the reticulocyte production index, which is related to erythrocytosis of the bone marrow. \%MICROr represents the proportion of reticulocytes less than $60 \mathrm{fL}$ in volume. $\mathrm{MCVm}$ represents the average volume of mature RBCs.

For better identification of MHA, we developed 2 new discrimination formulas using the linear discriminant analysis (LDA) method based on the IDA and $\alpha$-TT groups or the IDA and $\beta$-TT groups.

In addition, another 8 erythrocyte discriminant functions defined by the authors of the original published formulas were calculated and verified in this study (see Table 1).

Biochemical assays of iron status [serum iron (SI), $\mathrm{SF}$, and unsaturated iron-binding capacity (UIBC)] were performed using standard methods on a BS $-2000 \mathrm{~m}$ (determined for SF) and BS-800 (determined for SI and UIBC) analyzer (Mindray Corporation, Shenzhen, China). The BS-2000m and BS-800 analyzers were calibrated, controlled, and maintained according to the manufacturer's recommendations. Hemoglobin electrophoresis was performed by capillary electrophoresis in a Capillary FP analyzer (Sebia, France). Genetic analysis was performed by PCR-RDB analysis ( $\alpha$-TT, $\beta$-TT, and point mutations) and gap-PCR analysis ( $\alpha$-TT, gene deletion).

\section{Statistical analysis}

Statistical software package SPSS (SPSS; Chicago, IL,
USA) version 23.0 for Windows was used for statistical analysis of the results. Descriptive statistics are presented as the mean \pm standard deviation (SD) for normally distributed variables, and other variables are presented as the median and interquartile distribution range. The KolmogorovSmirnov test was used to assess the normality of data distributions. The Kruskal-Wallis test was performed for multiple comparisons among groups. Continuous variables conforming to a normal distribution were compared using the independent samples $t$-test. For non-normally distributed variables, the nonparametric Mann-Whitney $U$ test was applied. Receiver operating characteristic (ROC) analysis was utilized to determine the diagnostic performance of the single parameters and new formulas studied for the differential diagnosis of $\beta$-TT from IDA and $\alpha$-TT from IDA. A P value $<0.05$ was considered to indicate statistical significance.

\section{Results}

\section{Hematological parameters compared among groups}

Table 2 presents the hematological parameters of the IDA, $\alpha$-TT, and $\beta$-TT groups. In the IDA group, MCV, MCH, and MCVm were significantly higher than in the $\alpha$-TT and $\beta$-TT groups $(\mathrm{P}<0.05)$, and $\mathrm{RBC}$, reticulocyte percentage (Ret\%), and RPI in the IDA group were significantly lower than in the $\alpha$-TT and $\beta$-TT groups $(\mathrm{P}<0.05)$. There was no significant difference between the $\beta$-TT and IDA groups in terms of the hemoglobin (HGB), hematocrit (HCT), or \%MICROr values $(\mathrm{P}>0.05)$. The HGB and HCT values in the IDA group were significantly lower than in the $\alpha$-TT group $(\mathrm{P}<0.05)$. The \%MICROr and $\mathrm{MCVm}$ values were significantly higher in the IDA group than in the $\alpha$-TT group $(\mathrm{P}<0.05)$. The reticulocyte number $($ Ret\#) and $\mathrm{RBC}$ distribution width coefficient of variation (RDW-CV) values were significantly lower in the IDA group than in the $\beta$-TT group $(\mathrm{P}<0.05)$.

\section{Diagnostic efficiency of single and new combined bematological parameters}

Table 3 presents the ROC curve analysis results of single and combined parameters. ROC curve analysis of single parameters with significant differences between the groups was performed. In differentiating $\alpha$-TT, RBC as a single parameter showed the highest diagnostic efficiency. However, the diagnostic performance of RBC was still very low, so 
Table 1 Discriminant formulas for distinguishing thalassemia trait (TT) from iron deficiency anemia (IDA) in patients with microcytic red blood cells (RBCs)

\begin{tabular}{lccc}
\hline Abbr. & DF (reference) & Calculation & Cut-off thala. \\
\hline E\&F & England \& Fraser (10) & MCV $-(5 \times \mathrm{Hb})-\mathrm{RBC}-3.4$ & $<0$ \\
MI & Mentzler (11) & MCV/RBC & $<13$ \\
Sriv & Srivastava (12) & MCH/RBC & $<3.8$ \\
S\&L & Shine \& Lal (13) & MCV $\times$ MCH & $<1530$ \\
RDWI & RDW index (14) & MCV $\times$ RDW/RBC & $<20$ \\
RI & Ricerca (15) & RDW/RBC & $<4.4$ \\
G\&K & Green \& King (16) & MCV $\times$ RDW/I00 Hb & $<65$ \\
Logit-P1 & Logit-P1 (31) & $-10.14+1.64 \times$ RBC $+0.85 \times$ Ret $\%+0.07 \times$ IRF & $<0.84$ \\
\hline
\end{tabular}

Abbr., abbreviation; DF, discriminant formula; thala., thalassemia.

Table 2 Hematological parameters in the iron deficiency anemia (IDA), $\beta$-thalassemia trait ( $\beta$-TT), and $\alpha$-thalassemia trait ( $\alpha$-TT) groups

\begin{tabular}{|c|c|c|c|c|c|c|}
\hline Parameters & IDA $(n=340)$ & $A-T T(n=66)$ & B-TT (n=48) & $\begin{array}{c}\text { Kruskal- } \\
\text { Wallis test }(P)\end{array}$ & IDA/ $\alpha-T T(P)$ & IDA/ $\beta-T T(P)$ \\
\hline Age & $29.2 \pm 4.1$ & $29.5 \pm 4.6$ & $28.7 \pm 4.0$ & 0.5797 & - & - \\
\hline First trimester & $5(1.5 \%)$ & 7 (10.6\%) & $4(8.3 \%)$ & $<0.01$ & $<0.025$ & $<0.025$ \\
\hline Second trimester & $70(20.6 \%)$ & $24(36.4 \%)$ & $10(20.8 \%)$ & & & \\
\hline $\operatorname{RBC}\left(10^{12} / \mathrm{L}\right)$ & $4.33 \pm 0.43$ & $4.60 \pm 0.52$ & $4.63 \pm 0.49$ & $<0.05$ & $<0.05^{\&}$ & $<0.05^{\&}$ \\
\hline HGB (g/L) & 99 (93.0-104) & $101.00(95.00-109.75)$ & 98.00 (91.75-105.25) & $<0.05$ & $<0.05^{\star}$ & $0.823^{*}$ \\
\hline HCT (\%) & $31.75(30.00-33.10)$ & $32.75(30.15-35.30)$ & 31.75 (30.38-33.38) & $<0.05$ & $<0.05^{\star}$ & $0.671^{*}$ \\
\hline MCV (fL) & 72.60 (69.20-78.20) & $70.85(67.80-73.40)$ & 68.75 (66.65-71.33) & $<0.05$ & $<0.05^{\star}$ & $<0.05^{\star}$ \\
\hline RDW-SD & $38.65(35.60-43.23)$ & $37.15(35.60-40.00)$ & $37.20(35.98-38.85)$ & 0.156 & - & - \\
\hline Ret\# (10%) & $2.14(1.85-2.49)$ & $2.13(1.78-2.61)$ & $2.56(2.09-3.05)$ & $<0.05$ & $0.848^{*}$ & $<0.05^{\star}$ \\
\hline Ret\% (\%) & $0.09(0.08-0.11)$ & $0.10(0.08-0.12)$ & $0.12(0.10-0.14)$ & $<0.05$ & $<0.05^{\star}$ & $<0.05^{*}$ \\
\hline RPI & $0.90(0.80-1.10)$ & $1.00(0.80-1.30)$ & $1.10(0.90-1.30)$ & $<0.05$ & $<0.05^{\star}$ & $<0.05^{\star}$ \\
\hline \%MICROr (\%) & 13.99 (8.67-26.29) & $7.58(4.11-11.27)$ & $12.42(8.32-18.52)$ & $<0.05$ & $<0.05^{\star}$ & $0.200^{*}$ \\
\hline MCVm (fL) & $65.69(61.85-70.78)$ & $64.76(60.48-67.63)$ & $62.75(60.28-64.90)$ & $<0.05$ & $0.061^{*}$ & $<0.05^{\star}$ \\
\hline
\end{tabular}

Data are presented as the mean \pm SD or median with the interquartile range in parentheses. $P$ values were assessed using the Kruskal-Wallis test $\left({ }^{\sharp}\right)$, independent samples $t$-test $(\&)$, or Mann-Whitney $U$ test $\left({ }^{*}\right)$. \#P<0.05 by Kruskal-Wallis test. ${ }^{*} P<0.05$ by MannWhitney $U$ test. \&P<0.05 by independent samples $t$-test. RBC, red blood cell; HGB, hemoglobin concentration; HCT, hematocrit; HGB, hemoglobin; MCV, mean corpuscular volume; $\mathrm{MCH}$, mean corpuscular hemoglobin; MCHC, mean corpuscular hemoglobin concentration; RDW-CV, red blood cell distribution width coefficient of variation; RDW-SD, red blood cell width standard deviation; Ret\#, reticulocyte number; Ret\%, reticulocyte percentage; RPI, reticulocyte production index; \%MICROr, the proportion of reticulocytes less than $60 \mathrm{fl}$ in volume; MCVm, mean corpuscular volume of mature red blood cells. 
Table 3 Sensitivity, specificity, and Youden index of single parameters according to optimum cut-off values in the iron deficiency anemia (IDA), $\beta$-thalassemia trait $(\beta$-TT), and $\alpha$-thalassemia trait $(\alpha$-TT) groups

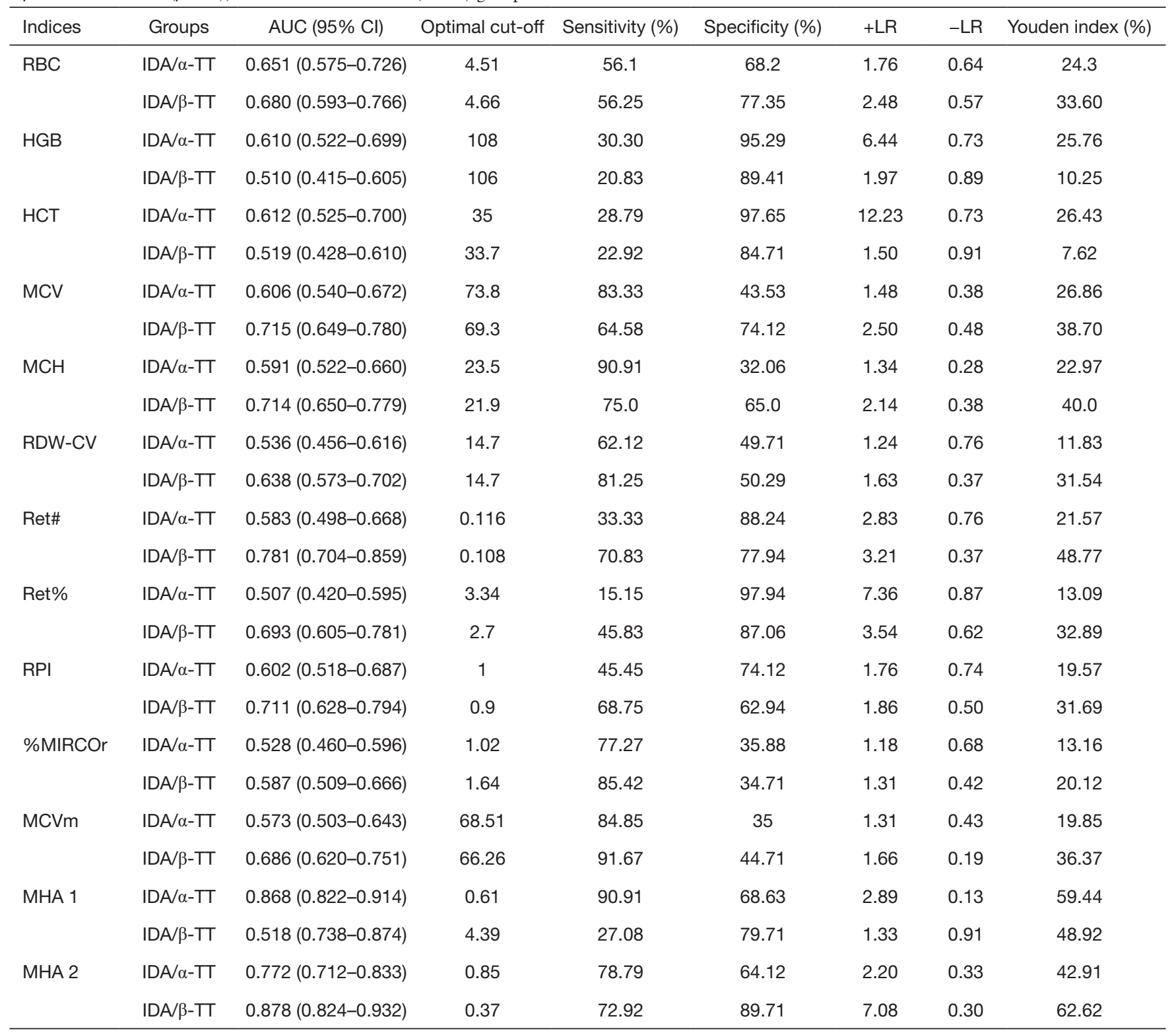

RBC, red blood cell; HGB, hemoglobin concentration; HCT, hematocrit; HGB, hemoglobin; MCV, mean corpuscular volume; MCH, mean corpuscular hemoglobin; $\mathrm{MCHC}$, mean corpuscular hemoglobin concentration; RDW-CV, red blood cell distribution width coefficient of variation; RDW-SD, red blood cell width standard deviation; Ret\#, reticulocyte number; Ret\%, reticulocyte percentage; RPI, reticulocyte production index; \%MICROr, the proportion of reticulocytes less than $60 \mathrm{fL}$ in volume; MCVm, mean corpuscular volume of mature red blood cell; MHA 1, formula for distinguishing $\alpha-T$ T carriers from IDA cases; MHA 2, formula for distinguishing $\beta$-TT carriers from IDA cases.

we developed a new combined parameter, MHA $1=0.42 \times$ $\mathrm{MCH}+0.08 \times \%$ MICROr $-0.57 \times$ RPI -9.38 , to differentiate $\alpha$-TT, which showed better diagnostic efficiency. The area under the ROC curve (AUC) of MHA 1 was 0.868 with a maximum Youden index (YI) of 59.44\% and an optimum cut-off value of 0.61 , and the corresponding sensitivity and specificity were $90.9 \%$ and $68.5 \%$, respectively (Figure $1 A$ ). Similarly, in differentiating $\beta$-TT, Ret\# as a single parameter showed the highest diagnostic efficiency. However, the diagnostic performance of Ret\# was still very low, so we 

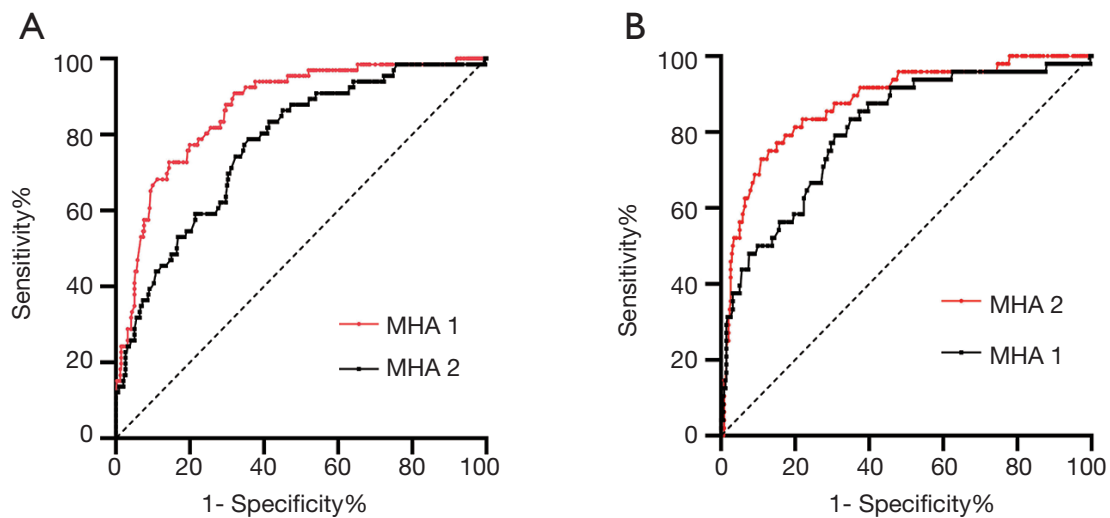

Figure 1 ROC curve analysis of MHA 1 and MHA 2. (A) ROC curve of MHA 1 for differentiating $\alpha$-thalassemia trait carriers (n=66) from iron deficiency anemia cases ( $n=340$ ), (B) ROC curve of MHA 2 for differentiating $\beta$-thalassemia trait carriers ( $\mathrm{n}=48$ ) from iron deficiency anemia cases ( $\mathrm{n}=340)$. Se., sensitivity; Sp., specificity; AUC, area under the curve; cut-off, optimum cut-off value; ROC, receiver operating characteristic; MHA, microcytic hypochromic anemia.

developed another new combined parameter, MHA 2 $=0.04 \times \%$ MICROr $+0.12 \times$ MCVm $-13.76 \times$ Ret\# -6.29, to differentiate $\beta$-TT, which showed better diagnostic efficiency. For MHA 2, the AUC was 0.878 with a maximum YI of $62.62 \%$ and an optimum cut-off value of 0.37 , and the corresponding sensitivity and specificity were $72.92 \%$ and $89.71 \%$, respectively (Figure $1 B$ ).

\section{Comparison of diagnostic efficiency with other reported indices}

The diagnostic performance of multiple other discriminant functions used to distinguish TT from IDA according to the proposed cut-off values reported in the literature are summarized in Table 4. Accordingly, the indices with the highest YI for distinguishing $\alpha$-TT from IDA are indicated by MHA 1 , and those for distinguishing $\beta$-TT from IDA are indicated by MHA 2. The discriminant efficiency of the Green and King (G\&K) index, RBC distribution width index (RDWI), and Ricerca index (RI) were significantly low.

The revised cut-off values of the formulas used to differentiate TT from IDA were determined. The sensitivity, specificity, and YI values according to the revised cutoff values are summarized in Table 5, and MHA 1 and MHA 2 were also determined for the comparison with other formulas. In differentiating $\alpha$-TT from IDA, the ranking of the formulas from the highest to the lowest according to their AUC values was MHA 1, MHA 2, Srivastava index, England and Fraser (E\&F) index, Mentzer index (MI), RI, RDWI, G\&K, Logit-P1, and Shine and Lal (S\&L) index.
The sensitivity, specificity, and YI of MHA 1 and MHA 2 were higher than those of the Srivastava index and MI (Table 5). In differentiating $\beta$-TT from IDA, the ranking of the formulas from highest to lowest according to their AUC values was MHA 2, MHA 1, Logit-P1, Srivastava index, S\&L, MI, E\&F, G\&K, RDWI, and RI. The sensitivity, specificity, and YI of MHA 1 and MHA 2 were higher than those of the other indices (Table 5). We found an MHA 1 of 0.61 to be optimal for distinguishing $\alpha$-TT from IDA, with $90.9 \%$ sensitivity and $68.6 \%$ specificity, resulting in a high AUC (0.868) and a high YI (59.4\%). The sensitivity and specificity of other discriminant functions are shown in Table 5. At the optimal cut-off, the MHA 1 AUC was significantly higher than the Srivastava index (0.66), whereas the YI was identical (27.25\%). However, MI had higher sensitivity and lower specificity than MHA 1 . The MI appeared to be very sensitive $(92.42 \%)$ but showed poor specificity (Table 5). The other discriminant functions had lower diagnostic performance (not shown).

\section{Discussion}

In general, iron deficiency, TT, anemia of chronic disease, and sideroblastic anemia are the four conditions that can cause microcytic anemia. The former two conditions are more common, particularly in outpatient populations in areas such as the Mediterranean basin and Southeast Asia. Regarding thalassemia disease in women, physiological changes during pregnancy worsen the severity of anemia and are significantly associated with an increased risk 
Table 4 Receiver operating characteristic (ROC) curve analysis of different discriminant formulas according to cut-off values in the literature in the iron deficiency anemia (IDA), $\beta$-thalassemia trait ( $\beta$-TT), and $\alpha$-thalassemia trait $(\alpha$-TT) groups

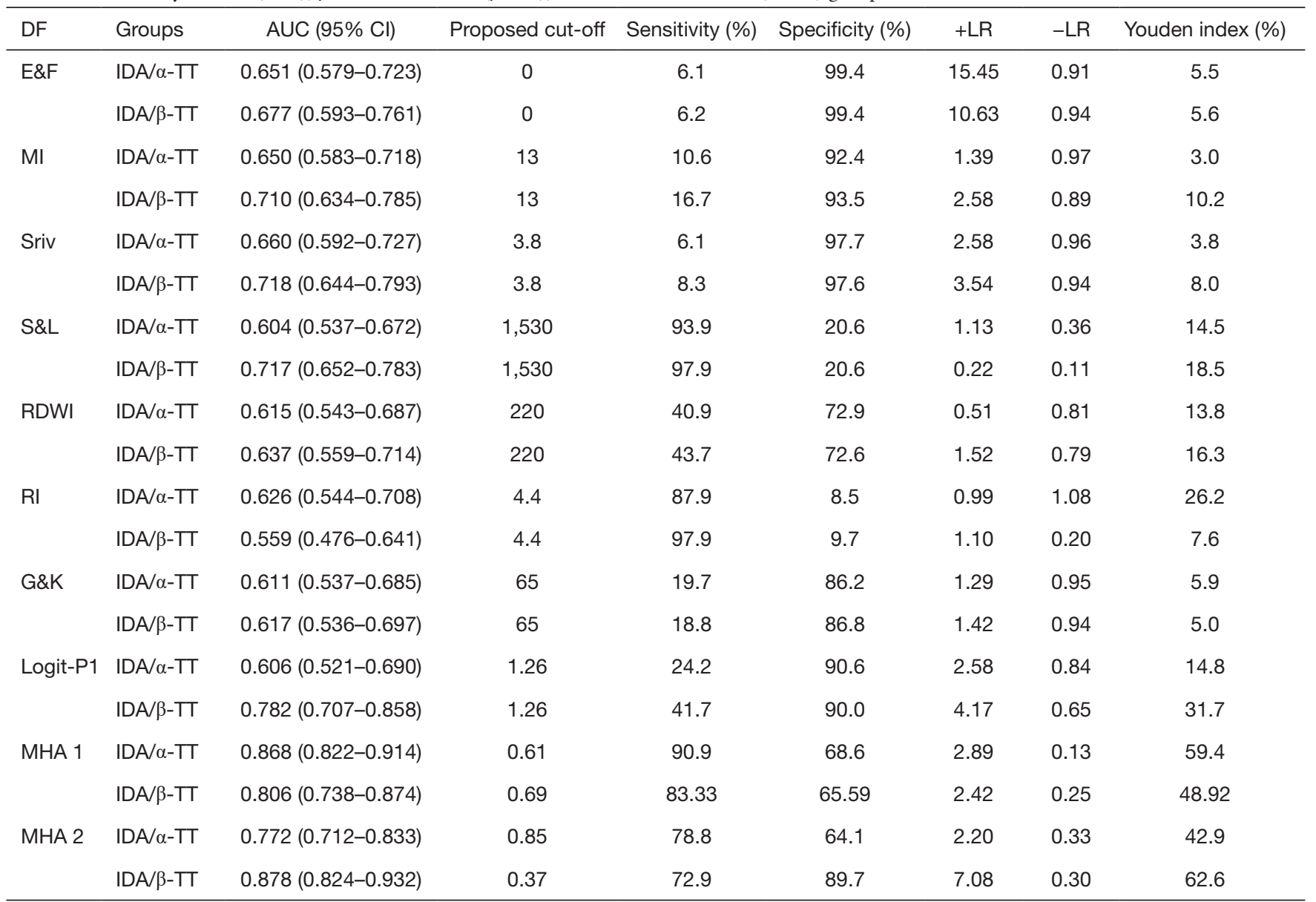

DF, discriminant formulas; AUC, area under the curve; Sensitivity, true positive/(true positive + false negative); Specificity, true negative/ (true negative + false positive); E\&F, England and Fraser Index; G\&K, Green and King Index; MI, Mentzer Index; RDWI, red blood cell distribution width index; S\&L, Shine and Lal Index; Srivastava, Srivastava Index.

of foetal growth restriction, preterm birth, and low birthweight. In pregnant women, early identification and correct classification have important implications for genetic counselling and proper treatment intervention $(32,33)$.

Red blood cell indices, and the various formulas derived from red blood cell parameters, have been proposed as simple, rapid, and inexpensive test methods that can be initially used to distinguish TT from IDA $(8,34,35)$. Thus, the corresponding indices show unsatisfactory applicative value. Studies addressing these characteristics in pregnant women are extremely limited, and their results are controversial.

In recent decades, reticulocyte parameters have been applied to screen for thalassemia more frequently, but few hematological formulas based on reticulocyte parameters have been designed to date. Thus, to screen out $\alpha$-TT or $\beta$-TT carriers from IDA cases, we designed two novel functions in our study to distinguish the $\alpha$-TT or $\beta$-TT carriers among microcytic anemic pregnant women. These formulas accounted for both erythrocyte and reticulocyte parameters rather than only erythrocyte parameters, as in the previous hematological indices. The two formulas demonstrated superior diagnostic performance compared to the previous hematological indices. It has been verified that erythrocytosis and severe microcytosis, together with a moderate increase in immature reticulocyte fraction (IRF), constitute the $\beta$-TT carrier profile, whereas anisocytosis and hypochromia correlate with IDA. RPI, \%MICROr, and 
Table 5 Receiver operating characteristic (ROC) curve analysis results and sensitivity, specificity, and Youden index of each discriminant formula according to revised cut-off values in the iron deficiency anemia (IDA), $\beta$-thalassemia trait ( $\beta$-TT), and $\alpha$-thalassemia trait ( $\alpha$-TT) groups

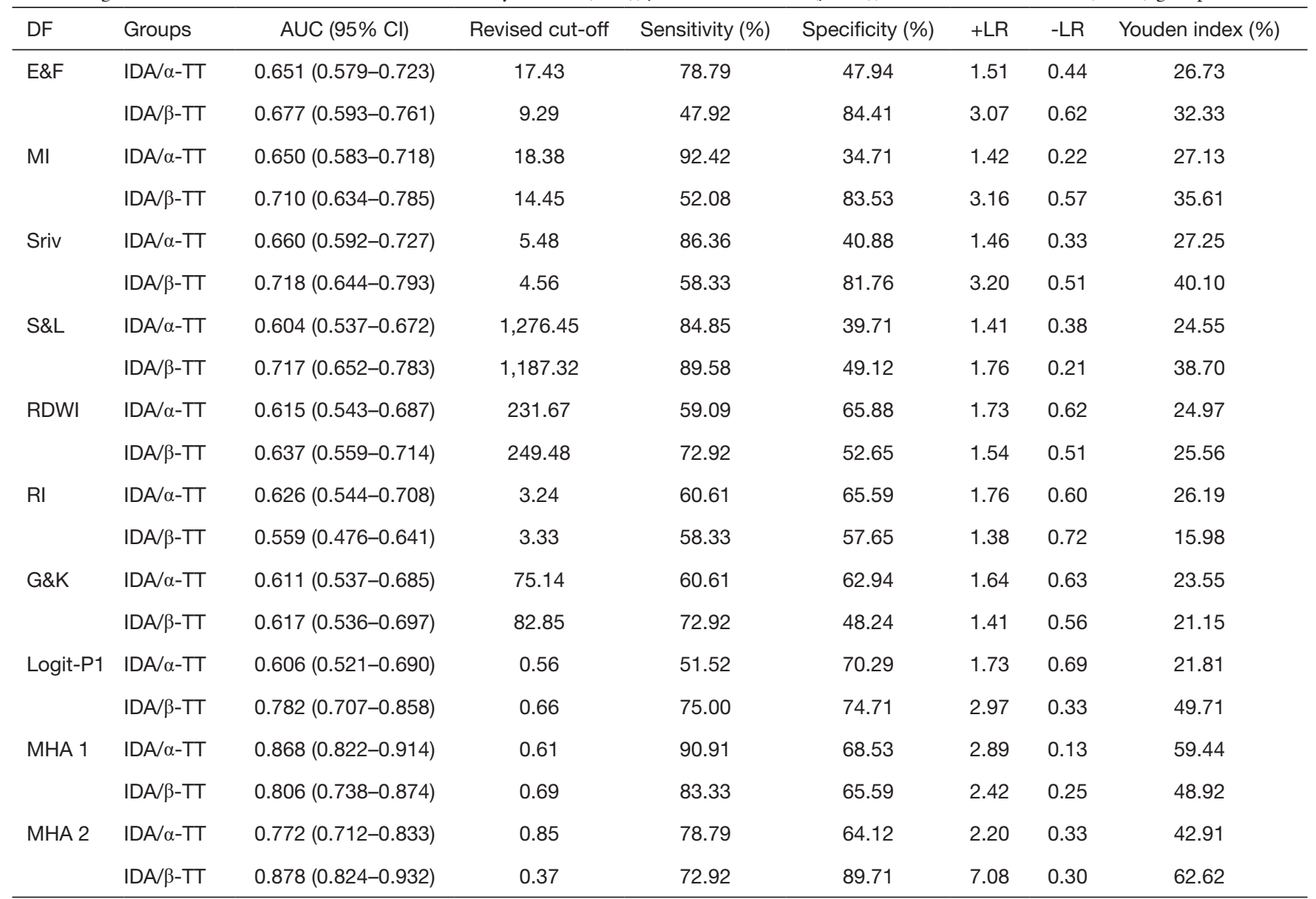

DF, discriminant formulas; AUC, area under the curve; Sensitivity, true positive/(true positive + false negative); Specificity, true negative/ (true negative + false positive); E\&F, England and Fraser Index; G\&K, Green and King Index; MI, Mentzer Index; RDWI, red blood cell distribution width index; S\&L, Shine and Lal Index; Srivastava, Srivastava Index.

$\mathrm{MCVm}$ were included in these equations, indicating the important clinical applicative value of reticulocyte-related parameters in distinguishing the two conditions. Formulas based only on erythrocyte parameters are not reflective of the important information of reticulocyte parameters. Therefore, simultaneous analysis of erythrocytes and reticulocytes can provide more detailed information to increase the differential diagnostic efficiency between TT and IDA.

Furthermore, the diagnosis of $\alpha$-TT and $\beta$-TT was made based on genetic analysis in our study, and all the TT carriers with concomitant IDA disease were excluded. Most hematological indices described in the previous literature were developed based on individuals diagnosed without genetic detection. This might be an important factor contributing to the differences in diagnostic performance between our investigation and others.

Recently, Logit-P1 was reported to be an optimal discriminator of TT from IDA in pregnant women, and the authors of the study derived a moderate AUC (0.760) with a sensitivity and specificity of $86.9 \%$ and $56.0 \%$, respectively (31). According to cut-off values reported in the previous literature and revised cut-off values, we assessed the reliability of $8 \mathrm{RBC}$ indices in distinguishing TT from IDA in our study. In addition, we evaluated two new formulas that we developed for the differential diagnosis of TT from IDA. The sensitivity and specificity values of MHA 1 and MHA 2 used in the differential diagnosis of $\alpha$-TT and IDA and $\beta$-TT and IDA were higher than those of the reported cut-off values in the literature 
(Table 5), demonstrating the much more useful of our new formulas for the differential diagnosis of TT and IDA in our patient population. The reliability of erythrocyte indices in the differential diagnosis of TT and IDA varies by the population. For example, in the Thai population, the Sirdah and Srivastava indices show the highest differential diagnostic efficacy, while in the Palestinian population, the Sirdah, G\&K, and RDWI show the highest differential diagnostic efficacy (8). Furthermore, in the Indian population, S\&L, Srivastava, and MI have been defined as the indices which show the highest differential diagnostic efficacy (36), and in the Chinese population, G\&F and E\&F show the highest differential diagnostic efficacy (9). These differences are due to the diversity of genetic mutations $(8,9,22)$. In addition, the sample size, the average age of the study group, and the heterogeneity of the included population may contribute to the perception of reliability of the different indices for different populations (22). If the definitive diagnosis is not accurate, for example, selecting subjects from a population of TT patients with concomitant IDA or selecting patients under iron deficiency treatment, the results may be inconsistent and misleading.

In our study, MHA 1 and MHA 2 were identified as the most reliable indices for the differential diagnosis of TT and IDA according to revised cut-off values. The differential diagnostic efficacy of the RI was lower than that of the other indices. Compared with the other 8 indices, the results of our study showed that MHA 1 had the highest sensitivity and specificity in differentiating $\alpha$-TT from IDA according to the revised cut-off values, and MHA 2 had the highest sensitivity and specificity in differentiating $\alpha$-TT from IDA according to the revised cut-off values.

The limitations of our study included the low number of patients with TT and the fact that the data were derived from a single center. Furthermore, we did not construct a validation group to verify our formulas in another selected group of pregnant women, so these results were based only on the data set of our present study. More clinical investigations in different populations should be performed to further assess the efficacy of these formulas.

In conclusion, erythrocyte or reticulocyte indices and formulas can be used as preliminary methods for the differential diagnosis of TT and IDA. According to our study results, MHA 1 and MHA 2 were the most useful indices for the differential diagnosis of $\alpha$-TT and IDA, and $\beta$-TT and IDA in pregnant women, whereas S\&L and RI were not as useful. Because cut-off values vary among populations, it would therefore be more beneficial for different populations to use these erythrocyte and reticulocyte indices or formulas to determine their own specific and appropriate cut-off values.

\section{Acknowledgments}

We thank all patients who participated in this study. We also wish to thank Mindray Corporation for providing the hematology analyzer and reagents for the measurement of erythrocyte and reticulocyte parameters. We thank engineer Jinxian Lin who installed, calibrated, and maintained the BC-6800Plus automated hematology analyzer and SC-120 auto slide maker \& stain machine. We also thank Ilouise B. from AJE (American Journal Experts) for critical revision of the manuscript.

Funding: This study was funded by the Shenzhen Municipal Government of China JCYJ20180703093402288.

\section{Footnote}

Reporting Checklist: The authors have completed the STARD reporting checklist. Available at http://dx.doi.org/10.21037/ atm-20-7896

Data Sharing Statement: Available at http://dx.doi. org/10.21037/atm-20-7896

Conflicts of Interest: All authors have completed the ICMJE uniform disclosure form (available at http://dx.doi. org/10.21037/atm-20-7896). All authors report grants from the Shenzhen Municipal Government of China, during the conduct of the study. The authors have no conflicts of interest to declare.

Ethical Statement: The authors are accountable for all aspects of the work in ensuring that questions related to the accuracy or integrity of any part of the work are appropriately investigated and resolved. This study was approved by Ethics Committee of Shenzhen Baoan Women's and Children's Hospital (LLSCHY 2019-10-1504), and informed consent was not required because only residual samples were collected and tested in this study. All procedures performed in this study involving human participants were in accordance with the Declaration of Helsinki (as revised in 2013).

Open Access Statement: This is an Open Access article distributed in accordance with the Creative Commons 
Attribution-NonCommercial-NoDerivs 4.0 International License (CC BY-NC-ND 4.0), which permits the noncommercial replication and distribution of the article with the strict proviso that no changes or edits are made and the original work is properly cited (including links to both the formal publication through the relevant DOI and the license). See: https://creativecommons.org/licenses/by-nc-nd/4.0/.

\section{References}

1. McLean E, Cogswell M, Egli I, et al. Worldwide prevalence of anaemia, WHO Vitamin and Mineral Nutrition Information System, 1993-2005. Public Health Nutrition 2009;12:444-54.

2. Goonewardene M, Shehata M, Hamad A. Anaemia in pregnancy. Best Pract Res Clin Obstet Gynaecol 2012;26:3-24.

3. Higgs DR, Engel JD, Stamatoyannopoulos G. Thalassaemia. Lancet 2012;379:373-83.

4. Weatherall DJ. Keynote address: The challenge of thalassemia for the developing countries. Ann N Y Acad Sci 2005;1054:11-7.

5. Lv W, Linpeng $\mathrm{S}, \mathrm{Li} Z$, et al. Non-invasive prenatal diagnosis for pregnancies at risk for $\beta$-thalassemia: a retrospective study. BJOG 2020. [Epub ahead of print].

6. Petrakos G, Andriopoulos P, Tsironi M. Pregnancy in women with thalassemia: challenges and solutions. Int $\mathrm{J}$ Womens Health 2016;8:441-51.

7. Urrechaga E, Hoffmann JJML. Critical appraisal of discriminant formulas for distinguishing thalassemia from iron deficiency in patients with microcytic anemia. Clin Chem Lab Med 2017;55:1582-91.

8. Pornprasert S, Panya A, Punyamung M, et al. Red cell indices and formulas used in differentiation of $\beta$-thalassemia trait from iron deficiency in Thai Adults. Hemoglobin 2014;38:258-61.

9. Shen C, Jiang YM, Shi H, et al. Evaluation of indices in differentiation between iron deficiency anemia and betathalassemia trait for Chinese children. J Pediatr Hematol Oncol 2010;32:e218-22.

10. England J M, Fraser P M. Differentiation of iron deficiency from thalassaemia trait by routine blood-count. Lancet 1973;1:449-52.

11. Mentzer W C J. Differentiation of iron deficiency from thalassaemia trait. Lancet 1973;1:882.

12. Srivastava PC. Differentiation of thalassaemia minor from iron deficiency. Lancet 1973;2:154-5.

13. Shine I, Lal S. A strategy to detect beta-thalassaemia minor. Lancet 1977;1:692-4.

14. Jayabose S, Giamelli J, Levondoglutugal O, et al. Differentiating iron deficiency anemia from thalassemia minor by using an RDW-based index. J Pediatr Hematol 1999;21:314.

15. Ricerca B M, Storti S, D'Onofrio G, et al. Differentiation of iron deficiency from thalassaemia trait: a new approach. Haematologica 1987;72:409-13.

16. Green R, King R. A new red cell discriminant incorporating volume dispersion for differentiating iron deficiency anemia from thalassemia minor. Blood Cells 1989;15:481.

17. Jahangiri M, Rahim F, Malehi AS. Diagnostic performance of hematological discrimination indices to discriminate between $\beta$ eta thalassemia trait and iron deficiency anemia and using cluster analysis: Introducing two new indices tested in Iranian population. Sci Rep 2019;9:18610.

18. Düzenli Kar Y, Özdemir ZC, Emir B, et al. Erythrocyte Indices as Differential Diagnostic Biomarkers of Iron Deficiency Anemia and Thalassemia. J Pediatr Hematol Oncol 2020;42:208-13.

19. Lian Y, Shi J, Nie N, et al. Reticulocyte Hemoglobin Equivalent (Ret-He) Combined with Red Blood Cell Distribution Width Has a Differentially Diagnostic Value for Thalassemia. Hemoglobin 2019;43:229-35.

20. Kabootarizadeh L, Jamshidnezhad A, Koohmareh Z. Differential Diagnosis of Iron-Deficiency Anemia from $\beta$-Thalassemia Trait Using an Intelligent Model in Comparison with Discriminant Indexes. Acta Inform Med 2019;27:78-84.

21. Piriyakhuntorn P, Tantiworawit A, Rattanathammethee T, et al. The role of red cell distribution width in the differential diagnosis of iron deficiency anemia and nontransfusiondependent thalassemia patients. Hematol Rep 2018;10:7605.

22. Hafeez Kandhro A, Shoombuatong W, Prachayasittikul V, et al. New Bioinformatics-Based Discrimination Formulas for Differentiation of Thalassemia Traits From Iron Deficiency Anemia. Lab Med 2017;48:230-7.

23. Kumar A, Saha D, Kini J, et al. The role of discriminant functions in screening beta thalassemia trait and iron deficiency anemia among laboratory samples. J Lab Physicians 2017;9:195-201.

24. Hoffmann JJ, Urrechaga E, Aguirre U. Discriminant indices for distinguishing thalassemia and iron deficiency in patients with microcytic anemia: a reply. Clin Chem Lab Med 2016;54:e107-8.

25. Hoffmann JJ, Urrechaga E Aguirre U. Discriminant indices for distinguishing thalassemia and iron deficiency 
in patients with microcytic anemia: a meta-analysis. Clin Chem Lab Med 2015;53:1883-94.

26. Huang TC, Wu YY, Chen YG Chen, et al. Discrimination index of microcytic anemia in young soldiers: a single institutional analysis. PLoS One 2015;10:e0114061.

27. $\mathrm{Ng} \mathrm{EH}$, Leung JH, Lau YS, et al. Evaluation of the new red cell parameters on Beckman Coulter DxH800 in distinguishing iron deficiency anaemia from thalassaemia trait. Int J Lab Hematol 2015;37:199-207.

28. Matos JF, Dusse LM, Stubbert RV, et al. Comparison of discriminative indices for iron deficiency anemia and $\beta$ thalassemia trait in a Brazilian population. Hematology 2013;18:169-74.

29. Nalbantoğlu B, Güzel S, Büyükyalçın V, et al. Indices used in differentiation of thalassemia trait from iron deficiency anemia in pediatric population: are they reliable? Pediatr Hematol Oncol 2012;29:472-8.

30. Centers for Disease Control (CDC). CDC criteria for anemia in children and childbearing-aged women. MMWR Morb Mortal Wkly Rep 1989;38:400-4.

31. Beyan C, Kaptan K, Ifran A. Predictive value of discrimination indices in differential diagnosis of iron

Cite this article as: Xiao $\mathrm{H}$, Wang $\mathrm{Y}$, Ye Y, Yang C, Wu X, Wu X, Zhang X, Li T, Xiao J, Zhuang L, Qi H, Wang F. Differential diagnosis of thalassemia and iron deficiency anemia in pregnant women using new formulas from multidimensional analysis of red blood cells. Ann Transl Med 2021;9(2):141. doi: 10.21037/atm-20-7896 deficiency anemia and beta-thalassemia trait. Eur J Haematol 2007;78:524-6.

32. Yang Y, Feng J, Peng B, et al. Combined detection of erythrocyte and reticulocyte to improve screening efficiency of thalassaemia trait in pregnancy. Frontiers Laboratory Med 2018;2:120-5.

33. Hanprasertpong T, Kor-anantakul O, Leetanaporn R, et al. Pregnancy outcomes amongst thalassemia traits. Arch Gynecol Obstet 2013;288:1051-4.

34. Charoenboon C, Jatavan P, Traisrisilp K, et al. Pregnancy outcomes among women with beta-thalassemia trait. Arch Gynecol Obstet 2016;293:771-4.

35. Dhara PT, Harsh AS. Discriminant functions in distinguishing beta-thalassemia trait and iron deficiency anemia: the value of the RDW-SD. J Internet J Hematol 2010;7:1-13.

36. Rathod DA, Amarjeet K, Patel V, et al. Usefulness of cell counter-based parameters and formulas in detection of beta-thalassemia trait in areas of high prevalence. Am J Clin Pathol 2007;128:585-9.

(English Language Editor: C. Betlazar-Maseh) 